\title{
Cerebral Mast Cells Participate In Postoperative Cognitive Dysfunction by Promoting Astrocyte Activation
}

\author{
Xiang Zhang ${ }^{\mathrm{a}}$ Hao Yao ${ }^{\mathrm{a}, \mathrm{b}}$ Qingqing Qian ${ }^{\mathrm{a}}$ Nana Lia Wenjie Jin ${ }^{\mathrm{a}}$ Yanning Qian ${ }^{\mathrm{a}}$ \\ ${ }^{\mathrm{a}}$ Department of Anesthesiology, the First Affiliated Hospital of Nanjing Medical University, ${ }^{\mathrm{b}}$ Cardiovascular \\ center, the Second Affiliated Hospital of Nanjing Medical University, Nanjing, P. R. China
}

\section{Key Words}

Mast cells - Astrocyte activation • Neuroinflammation - Postoperative cognitive dysfunction - Cromolyn

\begin{abstract}
Background: Astrocytes, the major glial cell type that has been increasingly recognized as contributing to neuroinflammation, are critical in the occurrence and development of postoperative cognitive dysfunction (POCD). Although emerging evidence showed that brain mast cells (MCs) are the "first responders" in neuroinflammation, little is known about the functional communication between MCs and astrocytes. Methods: In this study, we investigated the potential regulation of astrocyte activation by MCs. Rats received an intracerebroventricular injection of Cromolyn (an MC stabilizer) or sterile saline 30 min before undergoing open tibial fracture surgery, and the levels of neuroinflammation and the degree of memory dysfunction were evaluated at 1 day and 3 days after surgery. In the in vitro study, the effect of activated MCs on astrocytes were further clarified. Results: Surgery increased the number of $\mathrm{MCs}$, the astrocyte activation and the production of inflammatory factors, and resulted in cognitive deficits. Site-directed pre-injection of Cromolyn can inhibit this effect. In the vitro study, the conditioned medium from C48/80-stimulated mast cells (P815) could induce primary astrocyte activation and subsequent production of inflammatory cytokines, which could be inhibited by Cromolyn. Conclusion: These findings indicate that activated MCs could trigger astrocyte activation, be involved in neuroinflammation and possibly contribute to POCD. Interactions between MCs and astrocytes could provide potential therapeutic targets for POCD.

(C) 2016 The Author(s)

Published by S. Karger AG, Basel
\end{abstract}

\section{Introduction}

Postoperative cognitive dysfunction (POCD), a complication that occurs after surgery, has recently gained more attention. POCD may exist over long durations of time and

X. Zhang, H. Yao and Q. Qian contributed equally to this study.

Prof. Yanning Qian

Department of Anesthesiology, the First Affiliated Hospital of Nanjing Medical University, Nanjing (P. R. China); E-Mail yanning_qian@163.com 


\section{Cellular Physiology Cell Physiol Biochem 2016;40:104-116 \begin{tabular}{l|l|l}
\cline { 2 - 3 } and BOI: 10.1159/000452528 & ( ) 2016 The Author(s). Published by S. Karger AG, Basel
\end{tabular} Published online: November 18, 2016 www.karger.com/cpb \\ Zhang et al.: MCs-Induced Astrocytes Activation Participates in POCD}

may evolve into an irreversible central nervous system (CNS) disease [1]. However, the pathological mechanisms involved in POCD remain unclear. Hence, it is important to unravel the neuropathogenesis of POCD. Recent studies have demonstrated that surgery-induced neuroinflammation is involved in POCD development [2-5]. The occurrence and propagation of neuroinflammation rely on the interactions between glia and immune cells. However, gliaimmune cell communication has not been comprehensively investigated.

Astrocytes, which are major glial cells, participate in all essential CNS functions, including neuronal survival and differentiation [6,7], energy metabolism, blood flow regulation, ion and water homeostasis, neurotransmission, adult neurogenesis, immune defence, etc. [8]. Astrocytes, as one of primary responders to cellular stress, perturbation, infection and injury of the CNS, can secrete a number of important cytokines that affect the surrounding cells, such as neurons, microglia, and astrocyte, leading to neuroinflammation, neurodegeneration, and apoptosis. For instance, cytokines such as TNF-a, IL-1 $\beta$, IL-6, and transforming growth factor- $\beta 1$ (TGF- $\beta 1$ ) can act as pro- or anti-inflammatory mediators. Compared to moderate astrocyte activation, which is crucial in the repair of brain injury due to the secretion of neurotrophic factors, a rapid, prolonged and severe process may cause an excessive inflammatory response, ultimately contributing to neuronal damage and even neuronal apoptosis, resulting in neurodegenerative diseases [9]. Hence, the inhibition of astrocyte activation may improve surgery-induced neuroinflammation and cognitive dysfunction.

Although astrocyte activation has been recognized to contribute to neuroinflammation, proinflammatory mediators released from other immune cells, such as mast cells (MCs), are also crucial players. MCs, famous for their role in allergic responses, are also often found in the CNS, especially along the blood vessels and leptomeninges $[10,11]$. Under various types of stress, MCs serve as important sources of several mediators, including proteases and vasoactive amines such as tryptase and histamine [12], which have been reported to induce astrocyte activation and cytokine production in vitro $[13,14]$. These inflammatory mediators released by MCs participate in the pathology of several neuroinflammatory diseases [15]. Furthermore, activated brain MCs can disrupt the blood-brain barrier and can recruit neutrophils and activated T cells to enter the CNS $[5,16]$. Several molecular mechanisms for potential interactions between MCs and astrocytes have been determined in vitro [17]. However, the exact effects of MCs on astrocytes are still unclear. Understanding the triggers of neuroinflammation is key in improving cognitive function after surgery. In this study, we hypothesized that activated brain MCs contribute to astrocyte activation and neuroinflammation, which may then cause cognitive dysfunction after surgery.

\section{Materials and Methods}

\section{In Vivo Studies}

Animals. Male adult Sprague-Dawley (S-D) rats, 8 weeks-old, weighting 200-250 g, were used in this in vivo experiment. All rats were housed in groups of five per cage under standard environmental conditions (12 h light/dark cycle, ambient temperature of $22.0 \pm 1.0$, and $40 \%$ humidity) during the experimental period. Food and water were available ad libitum. The experimental process was approved by the Nanjing Medical University Animal Care and Use Committee. All experimental procedures involving animals were approved by IACUC (Institutional Animal Care and Use Committee of Nanjing Medical University).

Intracerebroventricular Cannula Implantation. Intracerebroventricular (i.c.v.) cannula implantation was used for the administration of drugs in rodents as previously described [4,5]. In brief, rats were placed in the stereotaxic apparatus (Stoelting Instruments, USA) for the i.c.v. administration of drugs following anaesthesia. According to the atlas of Paxinos and Watson (1982), guide cannulas (Plastic One) were inserted into the lateral ventricle $(0.8 \mathrm{~mm}$ posterior, $1.5 \mathrm{~mm}$ lateral, and $3.7 \mathrm{~mm}$ ventral to the bregma) and were secured to the skull with dental cement. The animals were allowed to recover in cages for one week. Animals with broken guide cannulas were removed from the procedure. At the time of drug administration, a corresponding injection cannula connected to a microsyringe pump by a PE-20 catheter was filled with drug 


\section{Cellular Physiology Cell Physiol Biochem 2016;40:104-116 \begin{tabular}{l|l|l}
\cline { 2 - 2 } DOI: 10.1159/000452528 & (C) 2016 The Author(s). Published by S. Karger AG, Basel
\end{tabular} and Biochemistry Published online: November 18, 2016 www.karger.com/cpb}

Zhang et al.: MCs-Induced Astrocytes Activation Participates in POCD

solution and was inserted into the guide cannula. The needle was maintained in this position for 5 min after the injection, and then it was slowly extracted from the brain.

\section{Surgery and Drug Administration}

The rats were randomly assigned to one of four groups with 12 rats in each group, and investigators were blinded to the experimental treatment: (A) i.c.v. injection of saline (Ctrl group); (B) i.c.v. injection of disodium cromoglycate (Cromolyn) (Cro group); (C) tibial fracture surgery following i.c.v. injection of saline (Sur group); or (D) tibial fracture surgery following i.c.v. injection of cromolyn (Cro+Sur group). Rats in the Cro group and Cro+Sur group received $200 \mu \mathrm{g}$ of Cromolyn $(100 \mu \mathrm{g} / \mu \mathrm{l}, 2 \mu \mathrm{l})$ i.c.v. $30 \mathrm{~min}$ before surgery, while the other animals received $2 \mu \mathrm{l}$ of saline.

Surgery consisted of the placement of an open tibia fracture of the left hind paw in aseptic conditions under anaesthesia as described previously [3-5]. Briefly, the left hind limb of the rat was meticulously shaved and disinfected with povidone iodine after the animals were placed under anaesthesia. Followed by the insertion of a $20-\mathrm{G}$ pin in the intramedullary canal, a middle incision was performed on the left hind paw; the periosteum was then stripped, and an osteotomy was performed. After the fracture, the wound was irrigated, and the skin was sutured. The animals were allowed to recover spontaneously from the anaesthesia. During the surgical process, the body temperature of the animals was monitored and maintained with the aid of warming pads. Analgesia ( $0.1 \mathrm{mg} / \mathrm{kg}$ buprenorphine) was given subcutaneously after the anaesthesia induction and before the skin incision.

\section{Behavioural tests}

Trace Fear Conditioning (TFC). To assess hippocampal-dependent memory, TFC was used in rodents as previously described $[4,5,18,19]$. Rats were trained to associate an environment (context) with a conditional stimulus (tone) and an unconditional stimulus (foot shock). Performance was assessed at 1 or 3 days after surgery. Freezing behaviour was recorded for $300 \mathrm{~s}$, and a decreased percentage of freezing time indicated an impairment of memory.

$Y$ maze test. The Y maze was another test that was used to assess learning ability as previously described. Nine continuous correct responses were defined as the learning criteria. The total number of stimulations to reach the criterion was recorded. All rats reached the learning criterion in the present study.

\section{MC staining and counting}

Rats were anaesthetized with $5 \%$ chloral hydrate $(0.8 \mathrm{ml} / 100 \mathrm{~g})$ and perfused first with $0.9 \%$ saline and then with cold $4 \%$ paraformaldehyde. The cerebral tissues were harvested and fixed with $4 \%$ paraformaldehyde at $4{ }^{\circ} \mathrm{C}$ for $24 \mathrm{~h}$. Brain sections (10- $\mu$ m thick) were prepared and were processed for toluidine blue (TB) staining and immunohistochemistry as follows. Slides with sections of the hippocampus were stained in $0.05 \%$ TB. The criteria for degranulation included the following: a loss of purple staining, a fuzzy appearance, a distorted shape, or multiple granules visible in the vicinity of the cell. The entire surface area of the CA1 area of the hippocampus was scanned manually using a light microscope at 200x magnification, and MCs were quantified with Cell D software (Olympus).

In addition, immunohistochemistry was performed to label MCs with a mast cell tryptase monoclonal antibody as follows.

\section{Immunohistochemistry}

Slides were incubated with the mast cell tryptase monoclonal antibody (1:100; Abcam; USA) or the GFAP monoclonal antibody (1:200; CST, USA) at $4{ }^{\circ} \mathrm{C}$ overnight and were then incubated with secondary antibody for $2 \mathrm{~h}$. Positive cells in the CA1 area of three hippocampus sections from each animal were visualized using a Leika 2500 microscope (Leica Microsystems, Wetzlar, Germany) at 200× magnification.

\section{In Vitro Studies}

P815 Cell Culture. P815 cells, an MC line derived from mouse tumour cells, were incubated as previously described [4]. Experiments were performed when the cells were in the logarithmic phase of growth.

Astrocyte-enriched cultures. Primary mouse astrocyte were prepared as described in a previous protocol, with slight modifications [20,21]. Briefly, whole brains were isolated from neonatal rats. The meninges and blood vessels were removed in cold PBS under a microscope. Next, the tissues were minced 


\section{Cellular Physiology Cell Physiol Biochem 2016;40:104-116 \begin{tabular}{l|l|l}
\hline DOI: 10.1159/000452528 & ( ) 2016 The Author(s). Published by S. Karger AG, Basel \\
\hline
\end{tabular} and Biochemistry Published online: November 18, 2016 www.karger.com/cpb}

Zhang et al.: MCs-Induced Astrocytes Activation Participates in POCD

with sterile scissors and were digested with $0.25 \%$ Trypsin-EDTA at $37^{\circ} \mathrm{C}$. Trypsinization was stopped by the addition of an equal volume of culture medium containing $10 \%$ FBS. The dissociated cells were passed through a $100-\mu \mathrm{m}$ pore mesh, pelleted at 1,500 rpm for 5 minutes, and re-suspended in culture medium. The cells were seeded onto poly-D-lysine pre-coated cell culture flasks and were cultured $37^{\circ} \mathrm{C}$ in a humidified atmosphere of 5\% CO2. The medium was replaced every three to four days after seeding. After the glial cells formed a confluent monolayer (10-14 days), the astrocyte cells were separated from the microglia by shaking. Cultures were passaged every 2 weeks into new $10-\mathrm{cm}$ dishes at least 3 times to achieve highly pure astrocyte cultures [22]. Culture purity was $>95 \%$, as determined by the assessment of GFAP expression.

Co-culture of astrocyte and P815 cells. After treatment with Cromolyn for $30 \mathrm{~min}$, the P815 cells $(1 \times$ 106 cells) were stimulated with Compound 48/80 (C48/80) and were cultured. Primary astrocytes (1× 106 cells) were grown in $5 \mathrm{~cm} \times 5 \mathrm{~cm}$ flasks until confluent and were cultured for 24 hours with conditioned medium from P815 cells that had undergone different treatments.

\section{ELISA}

The concentrations of tumour necrosis factor- $\alpha$ (TNF- $\alpha$ ) and interleukin-6 (IL-6) in rat hippocampus tissue extracts and in the culture medium of astrocyte were measured with a commercial ELISA kit from R\&D Systems. The concentrations of histamine and mast cell tryptase in the supernatant of MCs were quantified with an ELISA kit from Fitzgerald.

Western Blotting

Hippocampal tissue extracts and astrocyte cells were collected and homogenized in RIPA lysis buffer (Biyuntian, Shanghai, China), which contained $50 \mathrm{mM}$ Tris (pH 7.4), $150 \mathrm{mM} \mathrm{NaCl}, 1 \%$ Triton X-100, 1\% sodium deoxycholate, $0.1 \%$ SDS, sodium orthovanadate, sodium fluoride, EDTA, leupeptin, etc. After an incubation for $20 \mathrm{~min}$ on ice, the lysate was centrifuged, and protein concentration was determined with a BCA kit. Proteins $(50 \mu \mathrm{g})$ were denatured with sodium dodecyl sulphate (SDS) sample buffer and separated by $10 \%$ SDS-polyacrylamide gel electrophoresis. Proteins were transferred to PVDF membranes (Millipore) with a Bio-Rad miniprotein-III wet transfer unit. The membranes were incubated with 5\% BSA dissolved in Tris-buffered saline with Tween 20 (TBST) (pH 7.5, $10 \mathrm{mM}$ Tris- $\mathrm{HCl}, 150 \mathrm{mM} \mathrm{NaCl}$, and 0.1\% Tween 20) at room temperature for $1 \mathrm{~h}$. This was followed by the incubation of the membranes in different antibody solutions overnight at $4^{\circ} \mathrm{C}$. The following primary antibodies were used: mouse monoclonal anti-GFAP (CST, \#3670), rabbit monoclonal anti-Tau (Abcam, ab32075), c-Jun N-terminal kinase (JNK, CST, \#9258S), phospho-JNK (CST, \#4668), Extracellular regulated protein kinases (ERK, CST, \#4695S), and phospho-ERK (CST, \#14227). After adding the goat-anti-mouse or goat-anti-rabbit secondary antibody (1:5000) for $1 \mathrm{~h}$, the protein bands on the membranes were detected with an enhanced chemiluminescence kit.

\section{RNA purification and Real-time PCR}

Total RNA extraction and real-time PCR amplification was performed as previously described. Primers included the following: mouse GAPDH forward, AAC TTT GGC ATT GTG GAA GG reverse, GGA TGC AGG GAT GAT GTT CT; mouse GFAP forward, 5'-CGG AGA CGC ATC ACC TCT G-3', and reverse, 5'-TGG AGG AGT CAT TCG AGA CAA-3'. The relative mRNA values were normalized to the GAPDH gene control values and were calculated using the comparative cycle threshold $(\Delta \Delta \mathrm{Ct})$ method.

\section{Statistical Analysis}

All values are presented as the mean \pm SD. The significance of the differences between control samples and samples treated with various drugs was determined by one-way ANOVA followed by the post-hoc least significant difference test. Differences were considered significant at $\mathrm{P}<0.05$.

\section{Results}

Cromolyn inhibited the increase of MC number in the hippocampus induced by tibial surgery.

To examine whether MCs were involved in surgery-induced neuroinflammation, we first quantified brain MCs in TB and mast cell tryptase-stained sections of the hippocampus at 1 


\section{Cellular Physiology Cell Physiol Biochem 2016;40:104-116 \begin{tabular}{ll|l} 
DOI: 10.1159/000452528 & 02016 The Author(s). Published by S. Karger AG, Basel \\
\hline
\end{tabular}

Fig. 1. Cromolyn inhibited the surgery-induced increase in MC number in the hippocampus. (A) Immunostaining was used to detect mast cell tryptase in the CA1 area of the hippocampus. Scale bar: $50 \mu \mathrm{m}$. (B) Mast cells were stained with toluidine blue (TB) in the CA1 area of the hippocampus. Scale bar: $50 \mu \mathrm{m}$. (C) Quantification of tryptasepositive cells in the CA1 area of the hippocampus. (D) Quantification of mast cells stained with TB. ${ }^{*} \mathrm{P}<0.05$, $* * \mathrm{P}<0.01$ vs. the control group. \#P $<0.05$, \#\#P $<0.01$ vs. the surgery group. Data are presented as the mean $\pm S D(n=6)$.

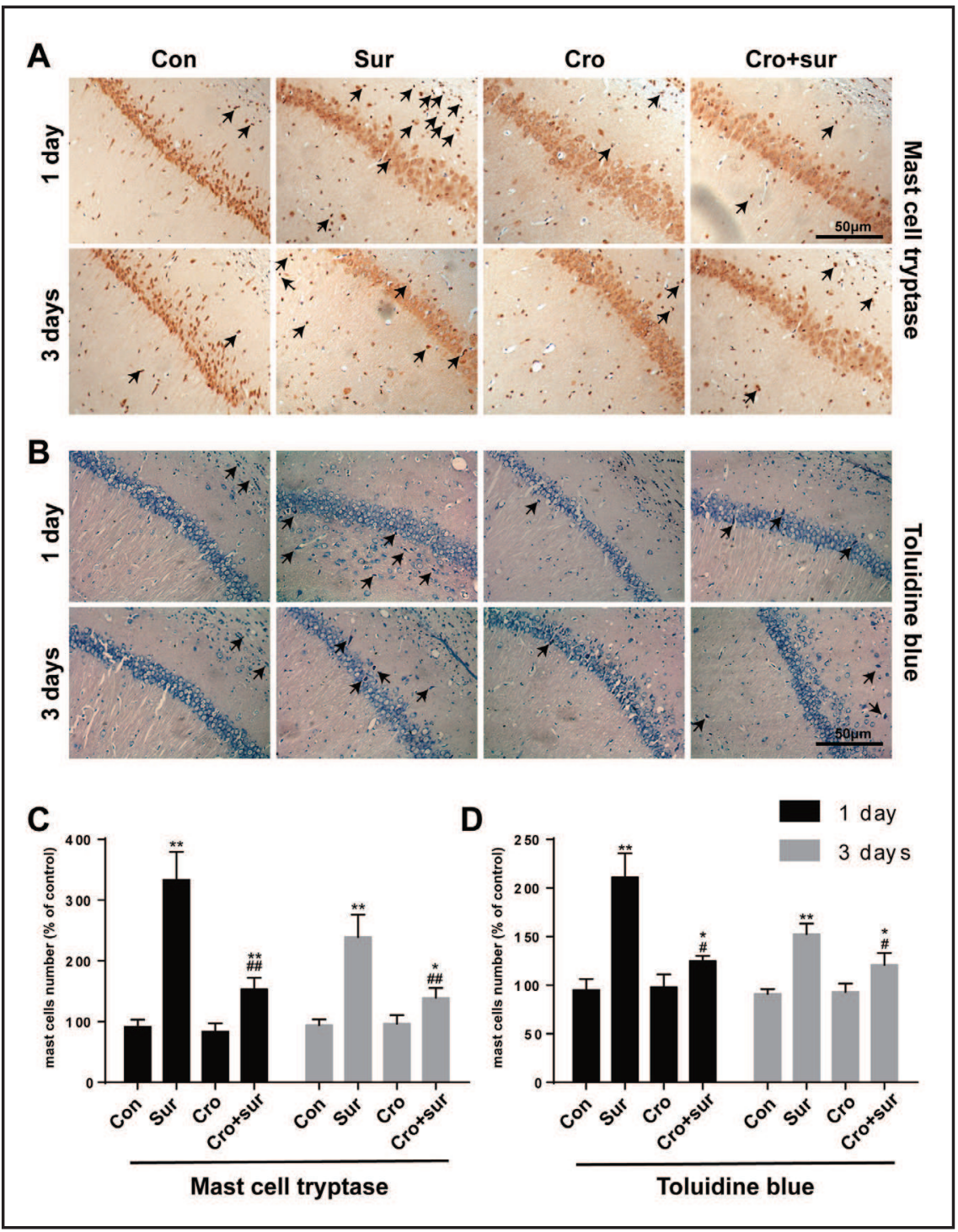

day and 3 days after surgery. As shown in Figures 1A and 1B, compared to the control group at 1 day and 3 days, surgery led to a significant increase in the number of MCs in the CA1 area of the hippocampus. This effect was inhibited by the i.c.v. injection of the MC stabilizer Cromolyn $(200 \mu \mathrm{g}, 2 \mu \mathrm{l})$. Treatment with Cromolyn alone had no effect on the number of MCs in the brain. These results suggest that surgery can induce MC activation in the hippocampus.

Cromolyn inhibited surgery-induced astrocyte activation and cytokine production in the hippocampus.

In order to explore the effects of activated MCs on astrocyte activation, immunostaining was used to detect GFAP, which is a marker for astrocytes. Surgery led to notable astrocyte activation in the hippocampus at 1 day and 3 days after surgery, as indicated by the large number of GFAP-positive cells (Figs. 2A and 2B) and the increased expression levels of GFAP protein (Fig. 4). The effect could once again be prevented through pre-treatment with the MC stabilizer Cromolyn. Cromolyn treatment alone had no effect on astrocyte activation in the brain.

Since astrocyte-mediated neuroinflammation is mainly due to the excessive secretion of proinflammatory factors from activated astrocytes and their downstream signalling cascades, the levels of the proinflammatory factors TNF- $\alpha$ and IL- 6 were detected by ELISA. 


\section{Cellular Physiology Cell Physiol Biochem 2016;40:104-116 \begin{tabular}{l|l|l}
\cline { 2 - 2 } DOI: 10.1159/000452528 & () 2016 The Author(s). Published by S. Karger AG, Basel
\end{tabular}

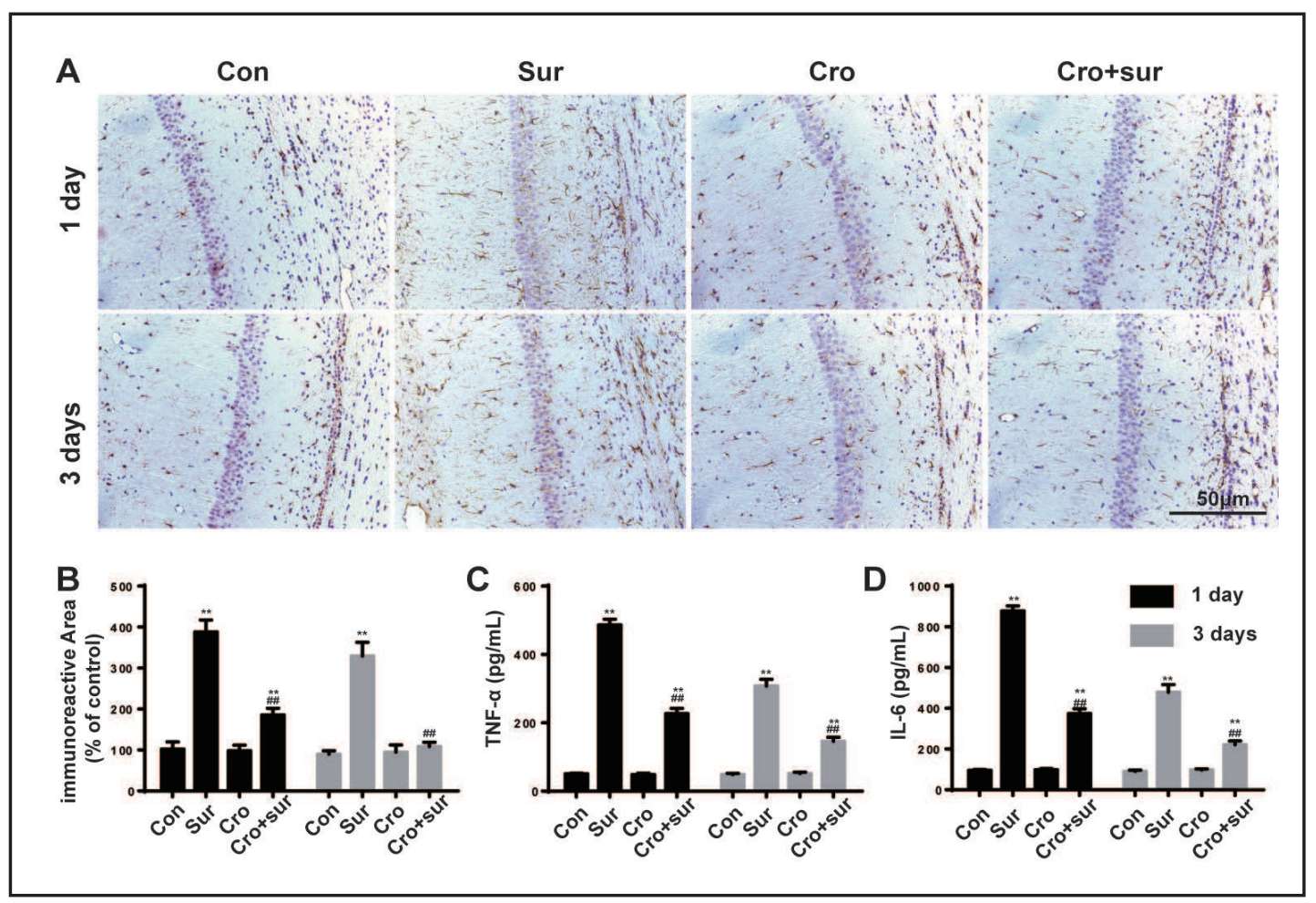

Fig. 2. Cromolyn inhibited the hippocampal astrocyte activation and cytokine production induced by tibial surgery. (A) Immunostaining was used to detect GFAP, a marker for astrocyte, in the CA1 area of the hippocampus. Scale bar: $50 \mu \mathrm{m}$. (B) Quantification of GFAP-positive cells in the CA1 area of the hippocampus. (C-D) The levels of proinflammatory factors TNF- $\alpha$ and IL- 6 were detected via ELISA. ${ }^{*} \mathrm{P}<0.05,{ }^{* *} \mathrm{P}<0.01 \mathrm{vs}$. the control group. $\# \mathrm{P}<0.05, \# \# \mathrm{P}<0.01$ vs. the surgery group. Data are presented as the mean $\pm \operatorname{SD}(\mathrm{n}=6)$.

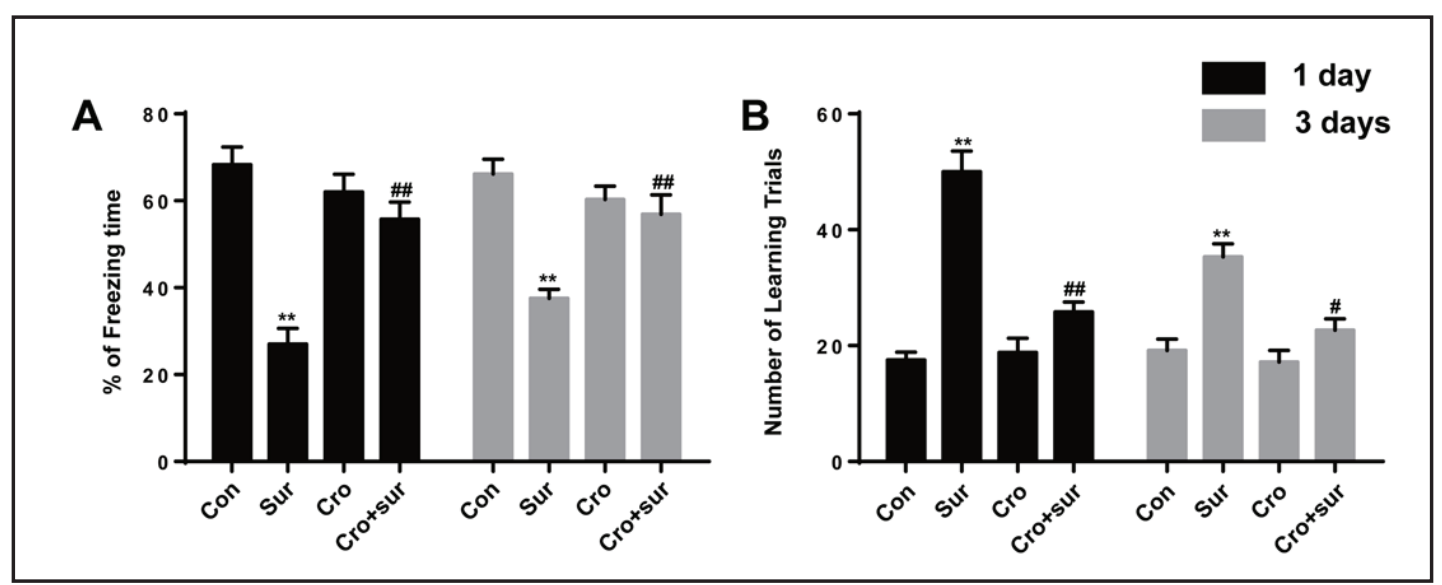

Fig. 3. Cromolyn treatment ameliorated surgery induced cognitive decline. (A) The freezing time in the Trace Fear Conditioning test and (B) the number of learning trials in the Y Maze test were recorded to analyse cognitive changes. ${ }^{*} \mathrm{P}<0.05,{ }^{* *} \mathrm{P}<0.01$ vs. the control group. $\# \mathrm{P}<0.05, \# \# \mathrm{P}<0.01$ vs. the surgery group. Data are presented as the mean $\pm \operatorname{SD}(n=10)$.

Surgery induced significant increases in TNF- $\alpha$ and IL- 6 production, and the injection of Cromolyn could inhibit the inflammatory response; Cromolyn alone had no effect on cytokine secretion (Figs. 2D and 2E). These results suggest that the stabilization of MCs can inhibit the activation of astrocyte and that it can reduce neuroinflammation.

\section{KARGER}




\section{Cellular Physiology \\ Cell Physiol Biochem 2016;40:104-116 and Biochemistry

Fig. 4. MAPK signalling was involved in surgery-induced astrocyte activation and neuroinflammation. (A) The phosphorylation levels of ERK and JNK, and the expression levels of GFAP and Tau in the hippocampus were detected via Western blotting using specific antibodies. Each blot is representative of three experiments. (B) The expression levels of GFAP, Tau, phosphorylated ERK and phosphorylated JNK were quantified and normalized to total levels. Each value was then expressed relative to the control, which was set to 1 . $* \mathrm{P}<0.05,{ }^{* *} \mathrm{P}<0.01$ vs. the control group. \#P $<0.05$, $\# \# \mathrm{P}<0.01$ vs. the surgery group. Data are presented as the mean $\pm \operatorname{SD}(n=5)$.

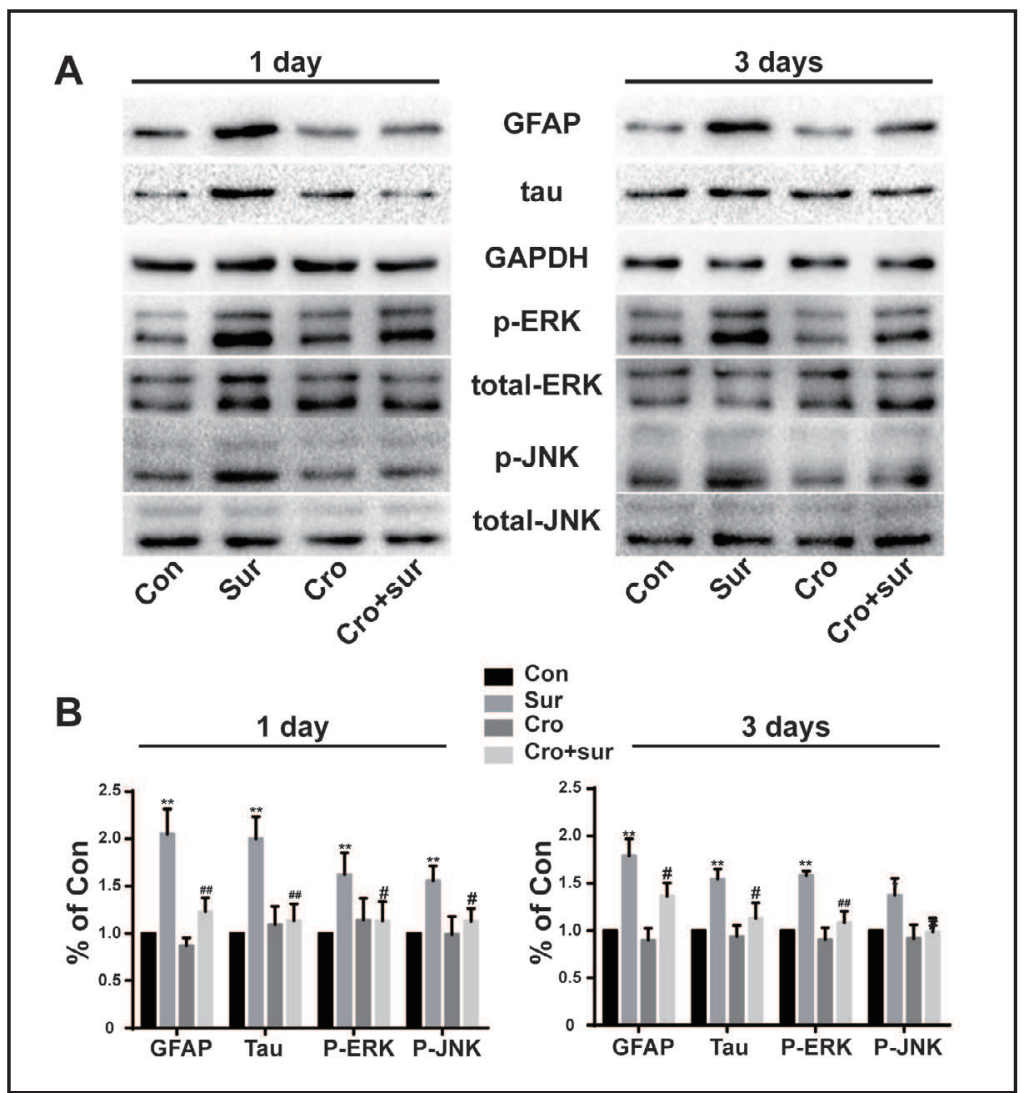

Cromolyn alleviated surgery-induced cognitive decline.

To further confirm whether activated mast cells participated in cognitive dysfunction, we evaluated spatial working memory with the TFC test and Y maze. Compared to the control groups, the rats that underwent surgery exhibited a significant cognitive impairment at 1 day and 3 days after surgery. Notably, treatment with Cromolyn significantly improved freezing behaviour and the number of learning trials. However, the injection of Cromolyn alone did not change the freezing time or the number of learning trials (Fig. 3A and 3B). These results suggest that Cromolyn may help protect against surgery-induced cognitive dysfunction.

Cromolyn inhibited surgery-induced MAPK activation in the hippocampus.

To confirm the signalling pathways involved in neuroinflammation, Tau and MAPK related proteins were evaluated with Western blot. Surgery led to increased levels of Tau and rapid phosphorylation of ERK and JNK in the hippocampus, a change that could be prevented by Cromolyn $(200 \mu \mathrm{g})$ pre-treatment (Fig. 4A and 4B). Cromolyn $(200 \mu \mathrm{g})$ alone had no obvious effect. These results suggest that the stabilization of MCs can inhibit the proinflammatory response in the hippocampus and that MAPK signalling pathways are involved in this process.

C48/80-activated P815 cells increased astrocyte activation.

Given that the data in vivo clearly indicated a relationship between MCs and neuroinflammation caused by surgery, we then sought to clarify the mechanism of this correlation. Since astrocyte activation is a recognized sign that triggers neuroinflammation in neurodegenerative disorders, we explored the effect of activated MCs on astrocytes in vitro.

At $24 \mathrm{~h}$ after C48/80 stimulation, the release of histamine and tryptase reached a peak, which was referred to as MC degranulation (Fig. 5). Astrocyte activation was detected via 


\section{Cellular Physiology Cell Physiol Biochem 2016;40:104-116 \begin{tabular}{l|l} 
DOI: 10.1159/000452528 & ( ) 2016 The Author(s). Published by S. Karger AG, Basel
\end{tabular}

Fig. 5. C48/80 evoked MC degranulation. Histamine (A) and mast cell tryptase (B) concentrations in the supernatant of P815 cells were evaluated to analyse the degree of activation and degranulation. ${ }^{*} \mathrm{P}<0.05$, $* * \mathrm{P}<0.01$ vs. the control group. Data are presented as the mean $\pm \operatorname{SD}(n=3)$.
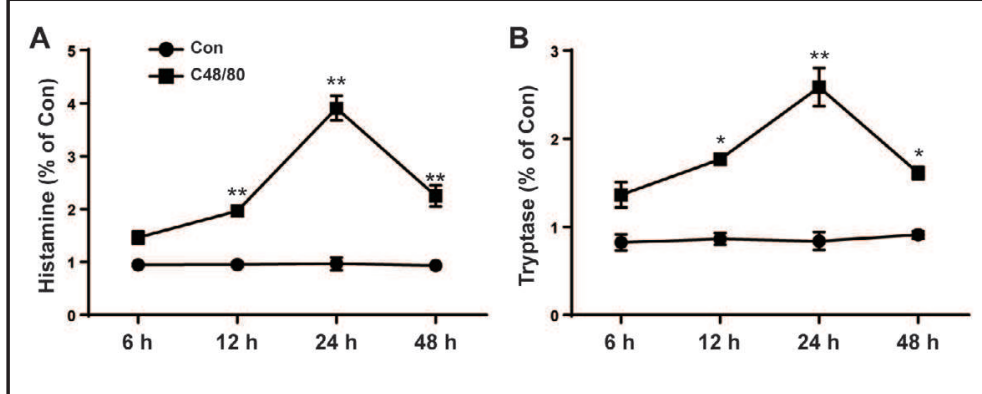

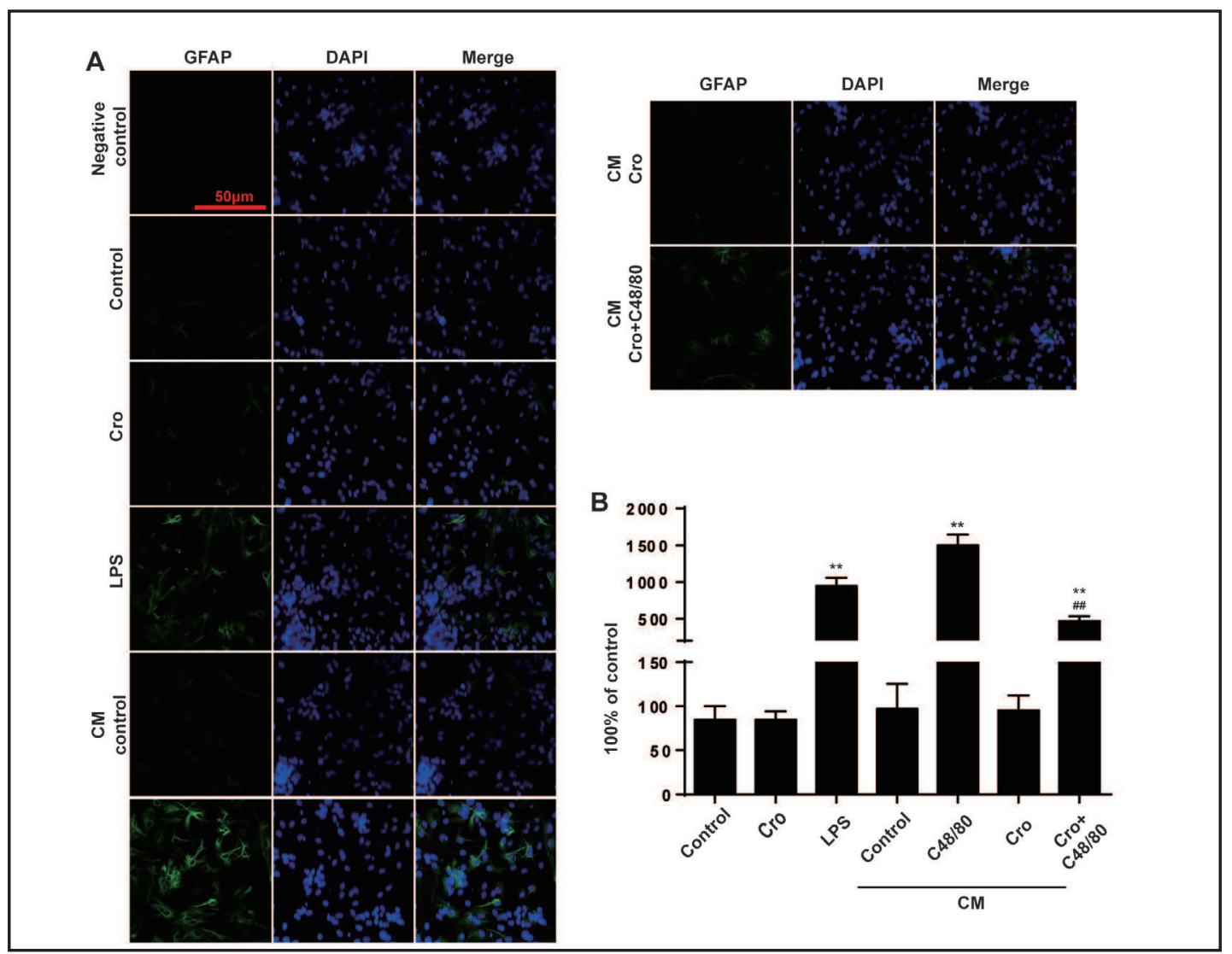

Fig. 6. Activated P815 cells increased astrocyte activation. (A) The cells were identified with a GFAP antibody and upregulated GFAP-immunopositive expression (green) on the activated astrocyte was observed using confocal scanning. The blue staining represents DAPI. Scale bar: $50 \mu \mathrm{m}$. (B) Quantification of GFAPpositive cells. ${ }^{*} \mathrm{P}<0.05$, ${ }^{* *} \mathrm{P}<0.01$ vs. the control group. $\# \mathrm{P}<0.05$, \#\#P $<0.01$ vs. the CM (P815 C48/80) group. Data are presented as the mean $\pm \mathrm{SD}(\mathrm{n}=3)$.

immunofluorescent staining, real-time PCR and Western blot for GFAP as before [21]. As shown in Figures 6 and 7A-C, similar to the effect of LPS exposure $(1 \mu \mathrm{g} / \mathrm{ml})$ alone, incubation with the conditional medium (CM) from P815 cells with C48/80-stimulation (24 h) for $24 \mathrm{~h}$ could induce astrocyte activation. Pre-treatment with Cromolyn $(10 \mu \mathrm{g} / \mathrm{ml})$ for $30 \mathrm{~min}$ could prevent the effect of MCs on astrocytes. These results indicated that mediators released by MC degranulation can induce astrocyte activation, which may be crucial in neuroinflammation.

MAPK signalling pathways were involved in astrocyte activation and cytokine production.

Since changes in the MAPK signalling pathways were observed during MC-induced astrocyte activation in vivo; we then investigated these signalling pathways in vitro for further 

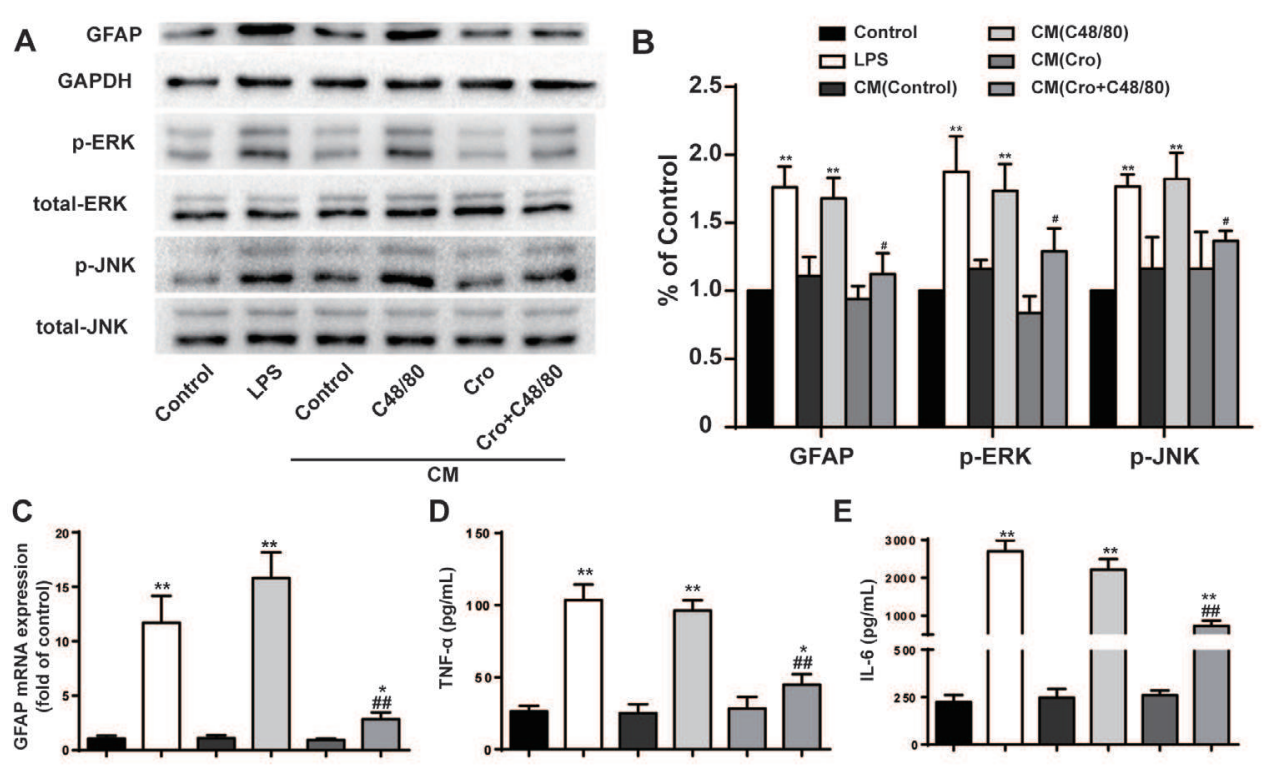

Fig. 7. MAPK signalling pathways were involved in astrocyte activation and the production of cytokines. (A) The expression levels of GFAP and the phosphorylation levels of ERK and JNK were detected via Western blotting using specific antibodies. Each blot is representative of three experiments. (B) Expression levels of GFAP, phosphorylated levels of ERK and phosphorylated levels of JNK were quantified and normalized to total levels. Each value was then expressed relative to the control, which was set to 1. (C) GFAP mRNA expression was detected via Real-Time PCR. (D-E) The levels of the proinflammatory factors TNF- $\alpha$ and IL- 6 were detected via ELISA. ${ }^{*} \mathrm{P}<0.05,{ }^{* *} \mathrm{P}<0.01$ vs. the control group. \#P $<0.05$, \#\#P $<0.01$ vs. the CM (P815 $\mathrm{C} 48 / 80)$ group. Data are presented as the mean $\pm \mathrm{SD}(\mathrm{n}=3)$.

verification. C48/80-stimulation ( $24 \mathrm{~h}$ ) of MCs in vitro increased the phosphorylation of ERK and JNK in astrocytes, which was ameliorated by Cromolyn $(10 \mu \mathrm{g} / \mathrm{ml})$ pre-treatment (Fig. 7A and 7B). To observe the inflammatory effects of MC-astrocyte co-cultures, TNF- $\alpha$ and IL-6 in the supernatant were detected via ELISA. As shown in Figures 7D and 7E, cytokine production was sharply upregulated by both the direct application of LPS and exposure to CM from P815 stimulated with C48/80; Cromolyn $(10 \mu \mathrm{g} / \mathrm{ml})$ pre-treatment could reduce the inflammatory response. Collectively, from this data, we concluded that MAPK signalling pathways were involved in MC-induced astrocyte activation and the subsequent production of cytokines.

\section{Discussion}

Pathological mechanisms underlying neuroinflammation have not been well characterized, even though neuroinflammation may contribute to the occurrence and development of POCD. Previous studies evaluated neuroinflammation by exploring glia and MCs in isolation from each other, ignoring the important role of the communication between them [23]. In this study, we explored the effects of activated MCs on astrocyte activation and the role of this interaction in POCD. We found that activated brain MCs can induce astrocyte activation and that it can aggravate neuroinflammation, resulting in cognitive dysfunction. These results may provide a novel therapeutic target for the treatment of POCD.

POCD has gained increasing attention due to the long-term adverse outcomes associated with the disorder, including a heavy economic burden, a low quality of life and an increasing mortality rate $[24,25]$. In rodents, surgery may induce deficits in exploratory behaviour and spatially-based working memory. TFC is widely used to assess learning 


\section{Cellular Physiology Cell Physiol Biochem 2016;40:104-116 \\ \begin{tabular}{ll|l} 
and Biochemistry Published online: November 18, 2016 & $\begin{array}{l}\text { ○ } 2016 \text { The Author(s). Published by S. Karger AG, Basel } \\
\text { www.karger.com/cpb }\end{array}$ \\
\hline
\end{tabular} \\ Zhang et al.: MCs-Induced Astrocytes Activation Participates in POCD}

and memory in rodents $[4,5,26]$. In the present study, we found that surgery can induce cognitive dysfunction in SD rats by decreasing the rate of freezing time on both 1 day and 3 days after surgery, which can be prevented by the i.c.v. administration of the MC stabilizer Cromolyn. These results were then confirmed with the Y maze test, one of the most widelyused paradigms for testing spatial based working memory in rodents [3-5, 27]. It is very sensitive for the measurement of early-period postoperative cognitive impairment and is usually used to assess hippocampus-dependent working memory [28, 29]. Hippocampal neurogenesis-related cognition is found to decline when animals are exposed to the electric shocks in the Y maze [30]. The results of the Y maze assessment were approximately the same as the results of the TFC. As observed in the results of our previous study, activated brain mast cells contributed to neuroinflammation and cognitive dysfunction by the promotion of BBB disruption [5], the activation of microglia and the induction of neuronal apoptosis [4]. Thus, we propose that activated MCs play a crucial role in the formation of cognitive deficits observed after tibial surgery. Brain MCs were implicated in the modulation of anxiety-like behaviour and provided the evidence for the behavioural importance of neuroimmune links [31]. The inhibition of the survival, migration and activity of MCs by masitinib can improve cognitive impairment in ADs [32]. In addition, approximately one-third of patients with mastocytosis display neuropsychological symptoms [33]. All of these findings show the closely correlation between brain MCs and cognitive function.

Astrocytes are crucial regulators of innate and adaptive immune responses in the injured central nervous system, and play primary roles in synaptic transmission and information processing by neural circuits, which are also considered to participate in neuroinflammation and CNS diseases [34-36]. Several experiments have shown that both microglia and astrocyte participate in neuroinflammation in animal models of neurodegeneration [37]. Microglial cells are mainly activated during the initial phase after minor surgery in rats, whereas the activation of astrocyte persists into the final periods [38]. Furthermore, astrocyte play a key role in the modulation of immune responses to CNS infections or disease [39]. Given that astrocyte reactivity was originally characterized by morphological changes (hypertrophy, remodelling processes) and the overexpression of the intermediate filament GFAP, we examined the level of GFAP to evaluate the activity of astrocytes. We found that surgery could induce astrocyte activation at 1 day and 3 days after surgery, which was accompanied by the secretion of proinflammatory cytokines. Therefore, the inhibition of astrocyte activation may offer clinical therapeutic benefits for neuroinflammation-related neurodegenerative disorders.

MCs are found in most tissues, including in the CNS, where they are often located adjacent to blood vessels and nerves [40] and can traverse the blood-brain barrier under pathological conditions [41]. Previous studies showed that activated brain MCs were the "first responders" in brain trauma and that they could induce microglial activation and neuronal apoptosis [4, 42]. Although other resident immune cells in the CNS also produce TNF- $\alpha$ (most notably microglia $[43,44]$, astrocyte $[13,21]$ and endothelial cells [45]), the presence and release of TNF- $\alpha$ from MCs precedes its detection in other cells. Our previous study also indicated that cerebral mast cells contribute to the pathogenesis of neuroinflammation and POCD in the rat by affecting the integrity of the BBB [5]. If the activation of brain MCs is necessary for the initiation of neuroinflammation and subsequent neurodegeneration, the inhibition of this response should be neuroprotective.

Even though astrocytes play a crucial role in neuroinflammation and chronic neurodegenerative diseases, the factors that are responsible for the over-activation of astrocytes are still unknown. Several studies have mentioned the potential crosstalk between MCs and astrocytes in vitro. There is evidence of the existence of H1- and H2-receptors on astrocytes in rats [14]. Patel et al reported that histamine could upregulate MMP-9 release via H1-receptor activation in astrocytes [46]. In addition, tryptase was shown to regulate the release of cytokines from astrocytes via PAR-2 signalling pathways in our previous study [13]. All of the above information notes the possibility of MC-astrocyte communication, which provides new insights for therapies targeting neuroinflammation by modulating the 


\section{Cellular Physiology Cell Physiol Biochem 2016;40:104-116

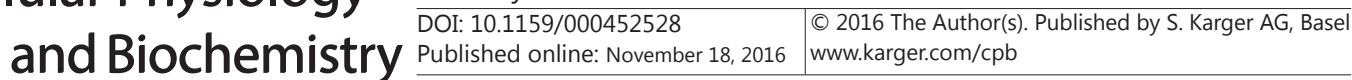 \\ Zhang et al.: MCs-Induced Astrocytes Activation Participates in POCD}

activation of MCs to control the over-activation of astrocytes. In this study, we found that surgery could activate not only MCs but also astrocyte at 1 day and 3 days after surgery and that the activation could be ameliorated by Cromolyn pre-treatment. To further clarify the effect of MCs on astrocytes directly, we co-cultured P815 cells and primary astrocytes in vitro. We found that C48/80-stimulated P815 cells can activate astrocyte and induce TNF- $\alpha$ and IL-6 secretion. Pre-treatment with the "mast cell stabilizer" Cromolyn for 30 min could inhibit this phenomenon. However, the P815 cells without C48/80-stimulation or with Cromolyn alone had no effect on astrocyte activation. The MAPK signalling pathway was involved. These results further confirm that activated MCs can induce astrocyte activation and cytokine production.

\section{Conclusion}

In summary, our findings demonstrate that peripheral surgery can induce MC degranulation, following by astrocyte activation and neuroinflammation, resulting in cognitive dysfunction, which can be improved by treatment with the "MC stabilizer" Cromolyn. In an in vitro study, we confirmed that only the activated MCs can induce astrocyte activation and that MAPK pathways are involved. These results indicate that activated MCs could contribute to neuroinflammation by evoking astrocyte over-activation and that MCastrocyte communication could provide a novel therapeutic target for neuroinflammationrelated diseases.

\section{Abbreviations}

POCD (postoperative cognitive dysfunction); MAPK (mitogen-Activated Protein Kinase); LPS (lipopolysaccharide); TNF- $\alpha$ (tumour necrosis factor- $\alpha$ ); IL-6 (interleukin-6); MCs (mast cells); Cromolyn (disodium cromoglycate); CNS (central nervous system); i.c.v. (intracerebroventricular); TFC (Trace Fear Conditioning); SDS (sodium dodecyl sulphate); TBST (Tris-buffered saline with Tween 20); JNK (c-Jun N-terminal kinase); ERK (extracellular regulated protein kinases); TB (toluidine blue).

\section{Acknowledgements}

We thank Prof. Shu Zhang (Clinical Research Center, the First Affiliated Hospital of Nanjing Medical University, 300 Guangzhou Road, Nanjing, Jiangsu 210029, P. R. China) for her guidance with experiments. This project was sponsored by the National Natural Science Foundation of China (No. 81400889, 81471410), and a Project Funded by the Priority Academic Program Development of Jiangsu Higher Education Institutions (PAPD).

\section{Disclosure Statement}

The authors declare that there are no conflicts of interest regarding the publication of this paper.

\section{References}

1 Moller JT, Cluitmans P, Rasmussen LS, Houx P, Rasmussen H, Canet J, Rabbitt P, Jolles J, Larsen K, Hanning CD, Langeron O, Johnson T, Lauven PM, Kristensen PA, Biedler A, van Beem H, Fraidakis O, Silverstein JH, Beneken JE, Gravenstein JS: Long-term postoperative cognitive dysfunction in the elderly ISPOCD1 study. ISPOCD investigators. International Study of Post-Operative Cognitive Dysfunction. Lancet 1998;351:857861. 


\section{Cellular Physiology Cell Physiol Biochem 2016;40:104-116 \begin{tabular}{ll|l}
\hline DOI: 10.1159/000452528 & $\begin{array}{l}\text { @ 2016 The Author(s). Published by S. Karger AG, Basel } \\
\text { www.karger.com/cpb }\end{array}$ \\
\hline
\end{tabular}}

Zhang et al.: MCs-Induced Astrocytes Activation Participates in POCD

2 Hovens IB, Schoemaker RG, van der Zee EA, Absalom AR, Heineman E, van Leeuwen BL: Postoperative cognitive dysfunction: Involvement of neuroinflammation and neuronal functioning. Brain Behav Immun 2014;38:202-210.

3 Lu SM, Yu CJ, Liu YH, Dong HQ, Zhang X, Zhang SS, Hu LQ, Zhang F, Qian YN, Gui B: S100A8 contributes to postoperative cognitive dysfunction in mice undergoing tibial fracture surgery by activating the TLR4/ MyD88 pathway. Brain Behav Immun 2015;44:221-234.

4 Zhang X, Dong H, Li N, Zhang S, Sun J, Zhang S, Qian Y: Activated brain mast cells contribute to postoperative cognitive dysfunction by evoking microglia activation and neuronal apoptosis. J Neuroinflamm 2016;13:127.

5 Zhang S, Dong H, Zhang X, Li N, Sun J, Qian Y: Cerebral mast cells contribute to postoperative cognitive dysfunction by promoting blood brain barrier disruption. Behav Brain Res 2016;298:158-166.

6 Raff MC, Barres BA, Burne JF, Coles HS, Ishizaki Y, Jacobson MD: Programmed cell death and the control of cell survival: Lessons from the nervous system. Science 1993;262:695-700.

7 Takeshima T, Shimoda K, Sauve Y, Commissiong JW: Astrocyte-dependent and -independent phases of the development and survival of rat embryonic day 14 mesencephalic, dopaminergic neurons in culture. Neuroscience 1994;60:809-823.

8 Oberheim NA, Goldman SA, Nedergaard M: Heterogeneity of astrocytic form and function. Methods Mol Biol 2012;814:23-45.

9 Okada S, Nakamura M, Katoh H, Miyao T, Shimazaki T, Ishii K, Yamane J, Yoshimura A, Iwamoto Y, Toyama Y, Okano H: Conditional ablation of Stat3 or Socs3 discloses a dual role for reactive astrocytes after spinal cord injury. Nat Med 2006;12:829-834.

10 Florenzano F, Bentivoglio M: Degranulation, density, and distribution of mast cells in the rat thalamus: A light and electron microscopic study in basal conditions and after intracerebroventricular administration of nerve growth factor. J Comp Neurol 2000;424:651-669.

11 Khalil M, Ronda J, Weintraub M, Jain K, Silver R, Silverman AJ: Brain mast cell relationship to neurovasculature during development. Brain Res 2007;1171:18-29.

12 Ikarashi Y, Yuzurihara M: Experimental anxiety induced by histaminergics in mast cell-deficient and congenitally normal mice. Pharmacol Biochem Behav 2002;72:437-441.

13 Zeng X, Zhang S, Xu L, Yang H, He S: Activation of protease-activated receptor 2-mediated signaling by mast cell tryptase modulates cytokine production in primary cultured astrocytes. Mediators Inflamm 2013;2013:140812.

14 Hosli L, Hosli E, Schneider U, Wiget W: Evidence for the existence of histamine H1- and H2-receptors on astrocytes of cultured rat central nervous system. Neurosci Lett 1984;48:287-291.

15 Theoharides TC, Cochrane DE: Critical role of mast cells in inflammatory diseases and the effect of acute stress. J Neuroimmunol 2004;146:1-12.

16 Sayed BA, Christy AL, Walker ME, Brown MA: Meningeal mast cells affect early T cell central nervous system infiltration and blood-brain barrier integrity through TNF: A role for neutrophil recruitment? J Immunol 2010;184:6891-6900.

17 Kim DY, Hong GU, Ro JY: Signal pathways in astrocytes activated by cross-talk between of astrocytes and mast cells through CD40-CD40L. J Neuroinflammation 2011;8:25.

18 Feng X, Degos V, Koch LG, Britton SL, Zhu Y, Vacas S, Terrando N, Nelson J, Su X, Maze M: Surgery results in exaggerated and persistent cognitive decline in a rat model of the Metabolic Syndrome. Anesthesiology 2013;118:1098-1105.

19 Almolda B, de Labra C, Barrera I, Gruart A, Delgado-Garcia JM, Villacampa N, Vilella A, Hofer MJ, Hidalgo J, Campbell IL, Gonzalez B, Castellano B: Alterations in microglial phenotype and hippocampal neuronal function in transgenic mice with astrocyte-targeted production of interleukin-10. Brain Behav Immun 2015;45:80-97.

20 Tarassishin L, Suh HS, Lee SC: LPS and IL-1 differentially activate mouse and human astrocytes: Role of CD14. Glia 2014;62:999-1013.

21 Li N, Zhang X, Dong H, Zhang S, Sun J, Qian Y: Lithium Ameliorates LPS-Induced Astrocytes Activation Partly via Inhibition of Toll-Like Receptor 4 Expression. Cell Physiol Biochem 2016;38:714-725.

22 Du F, Qian ZM, Zhu L, Wu XM, Qian C, Chan R, Ke Y: Purity, cell viability, expression of GFAP and bystin in astrocytes cultured by different procedures. J Cell Biochem 2010;109:30-37.

23 Skaper SD, Facci L, Giusti P: Mast cells, glia and neuroinflammation: Partners in crime? Immunology 2014;141:314-327. 


\section{Cellular Physiology Cell Physiol Biochem 2016;40:104-116 \begin{tabular}{ll|l} 
DOI: 10.1159/000452528 & $\begin{array}{l}\text { ○ 2016 The Author(s). Published by S. Karger AG, Basel } \\
\text { www.karger.com/cpb }\end{array}$ \\
\hline
\end{tabular}}

Zhang et al.: MCs-Induced Astrocytes Activation Participates in POCD

24 Monk TG, Weldon BC, Garvan CW, Dede DE, van der Aa MT, Heilman KM, Gravenstein JS: Predictors of cognitive dysfunction after major noncardiac surgery. Anesthesiology 2008;108:18-30.

25 Steinmetz J, Christensen KB, Lund T, Lohse N, Rasmussen LS: Long-term consequences of postoperative cognitive dysfunction. Anesthesiology 2009;110:548-555.

26 Gambus PL, Troconiz IF, Feng X, Gimenez-Mila M, Mellado R, Degos V, Vacas S, Maze M: Relation between acute and long-term cognitive decline after surgery: Influence of metabolic syndrome. Brain Behav Immun 2015;50:203-208.

27 Webster SJ, Bachstetter AD, Nelson PT, Schmitt FA, Van Eldik LJ: Using mice to model Alzheimer's dementia: An overview of the clinical disease and the preclinical behavioral changes in 10 mouse models. Front Genet 2014;5:88.

28 Hu N, Guo D, Wang H, Xie K, Wang C, Li Y, Wang C, Wang C, Yu Y, Wang G: Involvement of the blood-brain barrier opening in cognitive decline in aged rats following orthopedic surgery and high concentration of sevoflurane inhalation. Brain Res 2014;1551:13-24.

29 Wan Y, Xu J, Ma D, Zeng Y, Cibelli M, Maze M: Postoperative impairment of cognitive function in rats: A possible role for cytokine-mediated inflammation in the hippocampus. Anesthesiology 2007;106:436-443.

30 Zhao N, Zhong C, Wang Y, Zhao Y, Gong N, Zhou G, Xu T, Hong Z: Impaired hippocampal neurogenesis is involved in cognitive dysfunction induced by thiamine deficiency at early pre-pathological lesion stage. Neurobiol Dis 2008;29:176-185.

31 Nautiyal KM, Ribeiro AC, Pfaff DW, Silver R: Brain mast cells link the immune system to anxiety-like behavior. Proc Natl Acad Sci U S A 2008;105:18053-18057.

32 Piette F, Belmin J, Vincent H, Schmidt N, Pariel S, Verny M, Marquis C, Mely J, Hugonot-Diener L, Kinet JP, Dubreuil P, Moussy A, Hermine O: Masitinib as an adjunct therapy for mild-to-moderate Alzheimer's disease: A randomised, placebo-controlled phase 2 trial. Alzheimers Res Ther 2011;3:16.

33 Moura DS, Georgin-Lavialle S, Gaillard R, Hermine O: Neuropsychological features of adult mastocytosis. Immunol Allergy Clin North Am 2014;34:407-422.

34 Sofroniew MV, Vinters HV: Astrocytes: Biology and pathology. Acta Neuropathol 2010;119:7-35.

35 Barres BA: The mystery and magic of glia: A perspective on their roles in health and disease. Neuron 2008;60:430-440.

36 Colombo E, Farina C: Astrocytes: Key regulators of neuroinflammation. Trends Immunol 2016;37:608-620.

37 Khandelwal PJ, Herman AM, Moussa CE: Inflammation in the early stages of neurodegenerative pathology. J Neuroimmunol 2011;238:1-11.

38 Rosczyk HA, Sparkman NL, Johnson RW: Neuroinflammation and cognitive function in aged mice following minor surgery. Exp Gerontol 2008;43:840-846.

39 Martorana F, Guidotti G, Brambilla L, Rossi D: Withaferin a inhibits nuclear Factor-kappaB-Dependent ProInflammatory and stress response pathways in the astrocytes. Neural Plast 2015;2015:381964.

40 Dropp JJ: Mast cells in mammalian brain. Acta Anat (Basel) 1976;94:1-21.

41 Silverman AJ, Sutherland AK, Wilhelm M, Silver R: Mast cells migrate from blood to brain. J Neurosci 2000;20:401-408.

42 Jin Y, Silverman AJ, Vannucci SJ: Mast cells are early responders after hypoxia-ischemia in immature rat brain. Stroke 2009;40:3107-3112.

43 Gregersen R, Lambertsen K, Finsen B: Microglia and macrophages are the major source of tumor necrosis factor in permanent middle cerebral artery occlusion in mice. J Cereb Blood Flow Metab 2000;20:53-65.

44 Lambertsen KL, Meldgaard M, Ladeby R, Finsen B: A quantitative study of microglial-macrophage synthesis of tumor necrosis factor during acute and late focal cerebral ischemia in mice. J Cereb Blood Flow Metab 2005;25:119-135.

45 Hallenbeck JM: The many faces of tumor necrosis factor in stroke. Nat Med 2002;8:1363-1368.

46 Patel A, Vasanthan V, Fu W, Fahlman RP, MacTavish D, Jhamandas JH: Histamine induces the production of matrix metalloproteinase-9 in human astrocytic cultures via H1-receptor subtype. Brain Struct Funct 2016;221:1845-1860. 